\title{
Generative Design for project optimization
}

\author{
Andrea Di Filippo \\ Department of \\ Civil Engineer \\ University of Salerno \\ Fisciano (SA), Italy \\ anddifilippo1@unisa.it
}

\author{
Marco Lombardi \\ Department of \\ Industrial Engineering \\ University of Salerno \\ Fisciano (SA), Italy \\ malombardi@unisa.it
}

\author{
Francesco Marongiu \\ Department of \\ Industrial Engineering \\ University of Salerno \\ Fisciano (SA), Italy \\ f.marongiu@studenti.unisa.it
}

\author{
Angelo Lorusso \\ Department of \\ Industrial Engineering \\ University of Salerno \\ Fisciano (SA), Italy \\ alorusso@unisa.it
}

\author{
Domenico Santaniello \\ Department of \\ Industrial Engineering \\ University of Salerno \\ Fisciano (SA), Italy \\ dsantaniello@unisa.it
}

\begin{abstract}
The present work concerns the applications of Generative Design in the Architectural sector: this represents an iterative process that involves an algorithm for the generation of a few outputs to satisfy certain constraints. Generative Design can offer advantages to traditional building design and the urban planning processes, given its capability to manage complexity by optimizing specifically preselected criteria while still at the drafting stage. The case study is a building currently under construction at the Valladolid Campus, and the objective is to analyse whether or not its design process has been exhaustive. The first step was to define inputs and variables depending on the ultimate goals. Successively it was time to set the boundaries for the outputs in terms of the different displayable and measurable solutions. Then the last step was to select the optimal solution. The tools employed are Formit for basic modelling, Revit for BIM modelling, Dynamo for visual programming, using graphs and nodes customizable through Python scripts. Specifically, for the Generative Design part and results displaying, Refinery used an Autodesk beta version software. Refinery implements a genetic algorithm with an NSGA-II optimization, a meta-heuristic algorithm for multi-objective optimization. Once the optimal solution has been determined, it is possible to import the results back into a Dynamo for Revit environment to complete the BIM modeling according to the Level of Geometry (LOG) and Information (LOI) required.
\end{abstract}

Generative Design; optimization; BIM; Parametric Design; Genetic Algorithm.

\section{INTRODUCTION}

Nowadays, in addition to the interest in an aesthetic architecture aimed at satisfying the tastes of their clients, designers are paying more attention and emphasis to the performance of the building (structure, environment, building, socio-economic and cultural, etc.) [1], [2]. This change in design approach leads designers to adopt new technologies to support innovation, changing the traditional 2D design paradigm. Thus, civil engineers and architects have started a process of contamination towards experiences from other fields by adopting and learning technology from industrial design and mechanical engineering, for example, in which performance plays a crucial role. This process leads to adopting new computational design methods through computer science methodologies, such as generative and parametric approaches or isomorphic surfaces. In addition, many changes have been resulted from the advent and spread of low-cost and non-commercial electronic devices. Such devices are introduced to improve the quality of life through services $[3],[4],[5]$ that aim to increase human comfort and security [6], [7],[8]. Despite some issues regarding security, such devices bring considerable benefit to the well-being of human life [9],[10],[11] by giving the possibility to build a digital twin of real environments [12].

Given these changes and the new technological perspective, this study explores the potential of a BIM environment integrated with new computational design methods in order to maximize design proposal opportunities. The work leverages generative design to identify alternatives in the early stages of design, and parametric algorithm and existing BIM tools for modification of chosen alternatives and change management during the more advanced stages of design through construction. The following sections will explain each feature in more detail.

Among the emerging systems for automating basic ideas, generative systems are helping designers to have different design solutions explored quickly, saving time and work, and evaluating the most significant number of alternatives to the chosen performance requirements. While generative tools help AEC designers immerse themselves in their designs, they otherwise fail to meet the basic principles of information modelling and desired data management. To address this problem, the answer has been Generative Design, as the development of space - generative - BIM environment that 
allows for creativity, fluidity, and flexibility in design, while making minimal changes to the standard design process. In fact, using such an integrated platform, information relevant to design requirements can be the system input, while design algorithms can generate the design output. Therefore, this platform integration can help designers to solve complex multicriteria design problems. The enhancement of creativity characterizes this approach by allowing designers to use the idea seeding technique [13], supporting the design process by generating problem-free alternative designs [14], through altering various design parameters, observing (and reflecting) on the results in real-time [15].

The present work has as its object the study of a new design model, through the aid of Generative Design, with a first theoretical contextualization and a subsequent practical application in order to describe the entire design process, from concept to model.

The study, in fact, has been approached from an engineering point of view through the development of an application case referred to a new university building being designed at the Valladolid Campus to analyse the completeness of the analysis modelling processes. This synthesis presents a study conducted to explore different specifications of starting requirements and various possibilities of integrating generative design algorithms with existing BIM platforms, considering the complete process, from input setup, process description to the result and related outputs.

The main objective of this paper is to develop a conceptual method of the Generative BIM platform, developed with Autodesk's Refinery program in beta version, to maximize the efficiency of design processes and outline a methodology for supporting BIM applications: thus, from the very early basic idea stages to the final detailed design stages.

\section{THE GENERATIVE DESIGN BASED APPROACH}

This section will explore the essential concepts that underlie the ability to understand how Generative Design works as applied to the Revit and Dynamo programs through Refinery.

\section{A. Background}

In the late 1970s, William J. Mitchell referred to Generative Design as a system able to generate potential solutions to a given problem. In the early 21 st century, Thomas Fischer, and Christiane Herr [16] then defined it as a design approach in which "during the design process the designer does not interact with materials and products directly but through a generative system of some model." For Christiane Herr [17], a generative system refers to computer-aided generative systems that architects or engineers generally develop, thus reflecting the uniqueness of architectural design problems. John Frazer [18] described Generative Design as the use of "virtual computer space in a manner analogous to evolutionary processes in nature"; similarly, Jeffrey Krause [19], [20] defined it as the development of "systems that can enhance, evolve, or design architectural structures, objects, or spaces more or less autonomously." For Andre Chaszar and Sam Conrad Joyce [21], Generative Design overcomes the shortcomings of traditional manual design methods by "leveraging computational power and addressing issues of speed and accuracy, as well as complexity" and increased inventiveness as it increases "the number of design variations "and" the range of variations, "which include "happy accidents," i.e., unexpected outcomes that positively affect the design process. Fakhri Bukhari [22] more recently limited the scope of Generative Design to the use of "algorithms to generate a set of different solutions from a given set of design goals and constraints." complexity" and increased inventiveness as it increases "the number of design variations "and" the range of variations, "which include "happy accidents," i.e., unexpected outcomes that positively affect the design process. Fakhri Bukhari [22] more recently limited the scope of Generative Design to the use of "algorithms to generate a set of different solutions from a given set of design goals and constraints."

\section{B. The Generative Design Definition}

A possible shared definition based on state of the art, then, can be to consider the generative approach as the "ability to produce or create something." Some authors define Generative Design as a design process that refers primarily to the evolution of techniques in creating and producing design solutions [23]. In contrast, others do not limit Generative Design to evolutionary processes, considering it a design approach based on algorithmic or rule-based processes that generate multiple and, possibly, complex solutions [24]. Considering these two perspectives, it is trivial to observe how the association of Generative Design to evolutionary processes is restricted. It excludes other methods that generate design and differentiate it from other terms, such as Parametric Design. Therefore, it can be understood as a design paradigm that uses more autonomous algorithmic descriptions than Parametric Design. After initiating the generative process, the Generative Design system executes the coded instructions iteratively to meet the chosen criteria. As a result, complex outputs can be generated even from simple algorithmic descriptions. In many cases, the algorithm is difficult to correlate with the generated product, making it difficult to predict the outcome from simply reading the algorithmic description; it is therefore supported by visual design programs to support design choices.

Generative Design can refer to any design process in which the designer uses a system, most likely through one or more software programs, to solve the design problem with some level of automation. Briefly, one can refer to Generative Design as a collaborative design process between humans and computers. During this process, the designer defines design parameters. The computer produces design studies (alternatives). These are only evaluated based on quantifiable objectives established by the designer, improving the results based on studies previously carried out and feedback from the designer himself. Finally, outputs are classified according to how closely they approximate the designer's original objectives.

Generative Design represents a design framework that combines computational computing and human creativity to achieve results that would otherwise be unseen and/or unimaginable. It involves integrating a geometric system, a set of measurable goals, and a system to automatically generate, evaluate, and evolve a diverse number of design options. 
This approach can offer advantages for design, such as managing complexity and optimizing for specific criteria; this concept of complexity includes a great deal of input from experience, current demands, and specific needs.

Thus, there is the possibility of combining solutions based on real data, structuring discussion among stakeholders on design features and project goals, providing transparency on design assumptions, and offering a preliminary "live model" to build a complete design.

The framework consists of three main components:

- the generation of a broad design space of possible solutions through a custom design based on a geometric system;

- the evaluation of each solution through measurable objectives;

- the evolution of design generations through evolutionary computation.

Generative Design is, therefore, a framework that can be used on different scales and is flexible: in fact, its field of application varies in a wide range of design problems and scales, from industrial components, where it found its first applications, to buildings and cities.

\section{Used Tools}

The tools used were: Formit for basic design; Revit for modelling in a BIM environment; Dynamo for visual spreadsheet processing with nodes, graphs, and customization in Python. For the more specific part of Generative Design and visualization of the results was used Refinery, a beta version of Autodesk. The latter, through a genetic algorithm, implements NSGA-II optimization, a metaheuristic algorithm for multiobjective optimization.

\section{The Proposed Approach}

In Generative Design, the workflow involves the following consequential steps:

- Creative: design options are generated by the system, using algorithms and parameters specified by the designer.

- Analysis: the designs generated in the previous step are now measured and/or analysed according to the degree to which they meet the goals defined by the designer.

- Ranking: design options are sorted or ranked based on the results of the analysis.

- Evolution: the process of ranking design options to identify in which direction the design should be developed or evolved.

- Exploration: the designer compares and explores the generated designs, inspecting both the geometry and the evaluation results.

- Integration: a preferred design option is chosen and integrated into the larger project or design work.

Each of these phases can be further broken down into definition, execution, and results phases, where the definition phase is the designer's responsibility. In contrast, the execution phase and results are computer developed. In the definition phase, the designer must first establish the generation algorithm: this is the logic that defines how designs are generated, including constraints and rules. This definition phase is vital to all phases of the Generative Design process, as the validity of the results relies on the quality of the designer's input at this stage. Once the algorithm and its parameters have been defined, the computer begins to process, which means it starts generating different design options. The solutions generated

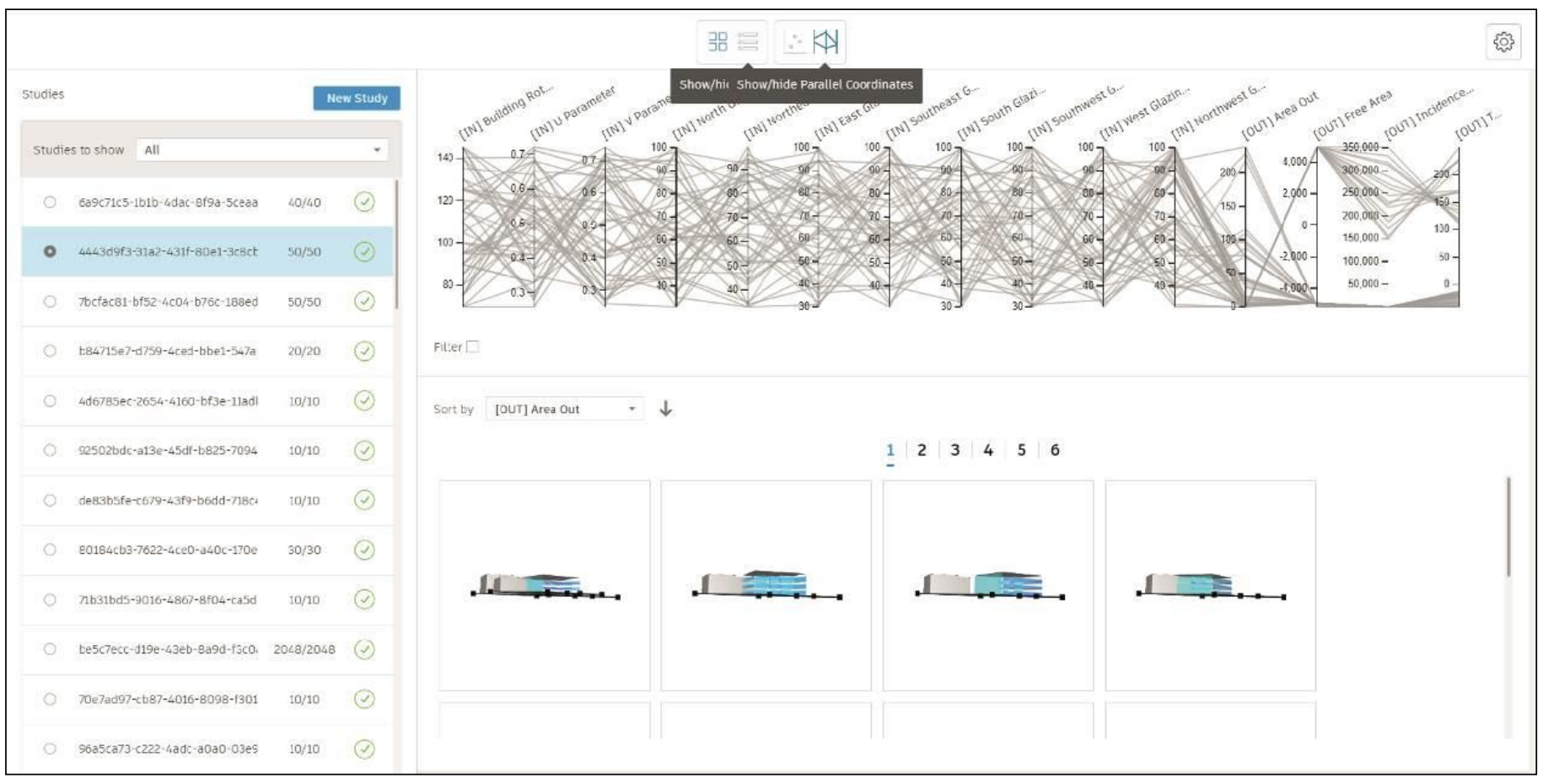

Fig. 1 System Output 
during the execution phase are the final outputs of each phase; used, then, as inputs or parameters in subsequent phases. For example, the designs created in the generation phase will be used as one of the input parameters in the analysis phase. (Fig.1)

So, the designer, in the problem definition phase, will need to start with the following basic data: work to be designed, the design parameters, conditions and needs to be met, what is not desired in the final design and what aspects are desired to maximize or minimize, etc. This phase will help the designer break down the problem into less complex sub-problems that will be the subject of analysis at each stage of the generative design process.

Underlying the entire Generative Design process is a multiobjective genetic algorithm. Generative algorithms are the logical paths that create new potential solutions in this approach. In other words, they are the engine of the algorithm: they give the rest of the program something to evaluate. Evaluator algorithms receive potential solutions from the generator and evaluate how good those options are. The evaluators must be mathematically specified in the design because they will have to provide a value that the algorithm must use to discriminate between solutions. These evaluators allow the user, or the program, to query each design option and choose the best one based on the aspects they wish to include. Solver algorithms can automatically execute a script multiple times, which may contain both generators and evaluators. Solvers typically require precise inputs. Optimize is the method of running an optimization function with Generative Design. During an optimization sequence, the design will be developed based on the results of the evaluator. The optimization process works by defining multiple "generations" (or iterations) of a design. Each iteration will use the input configurations from the previous generation to optimize the new design options. [25] Other types of solvers are: Randomize, Like This, and CrossProduct.

\section{CASE OF STUDY}

The case analysed is the design of the new structure of the Faculty of Architecture within the Valladolid Campus.

The study consists of two phases: the first involves designing the building volume in principle, the second focuses on the positioning and consequent study of radiation in the predetermined site. The study, therefore, begins with the design from the volumetric point of view of the building in question; in principle, it was thought to separate it into three buildings connected to each other, diversifying the functions into classrooms, laboratories, and offices, considering the needs of the users of the structure. In the Dynamo environment, is first set the basis of the generative process by identifying the inputs and constraints. In this case, the inputs go to define the dimensional base of the volumes. Instead, the starting constraints delimit the inter-floor heights of each building.

Once defined the starting points of the process, it was 'build' the connections of Dynamo to obtain the desired volumes and then set the outputs necessary for our results.

Defined the part of the graph inherent in the design of the volume, we start with the second phase of problem definition, inherent in positioning the volume and the radiation of the same. For this purpose, the position of a selected mass within the site boundary will be moved and rotated. Therefore, the solar incidence expressed concerning the area can be maximized or minimized.

This latter workflow relies heavily on Dynamo's "Solar Analysis" node, making external hyperlinks to a web service to

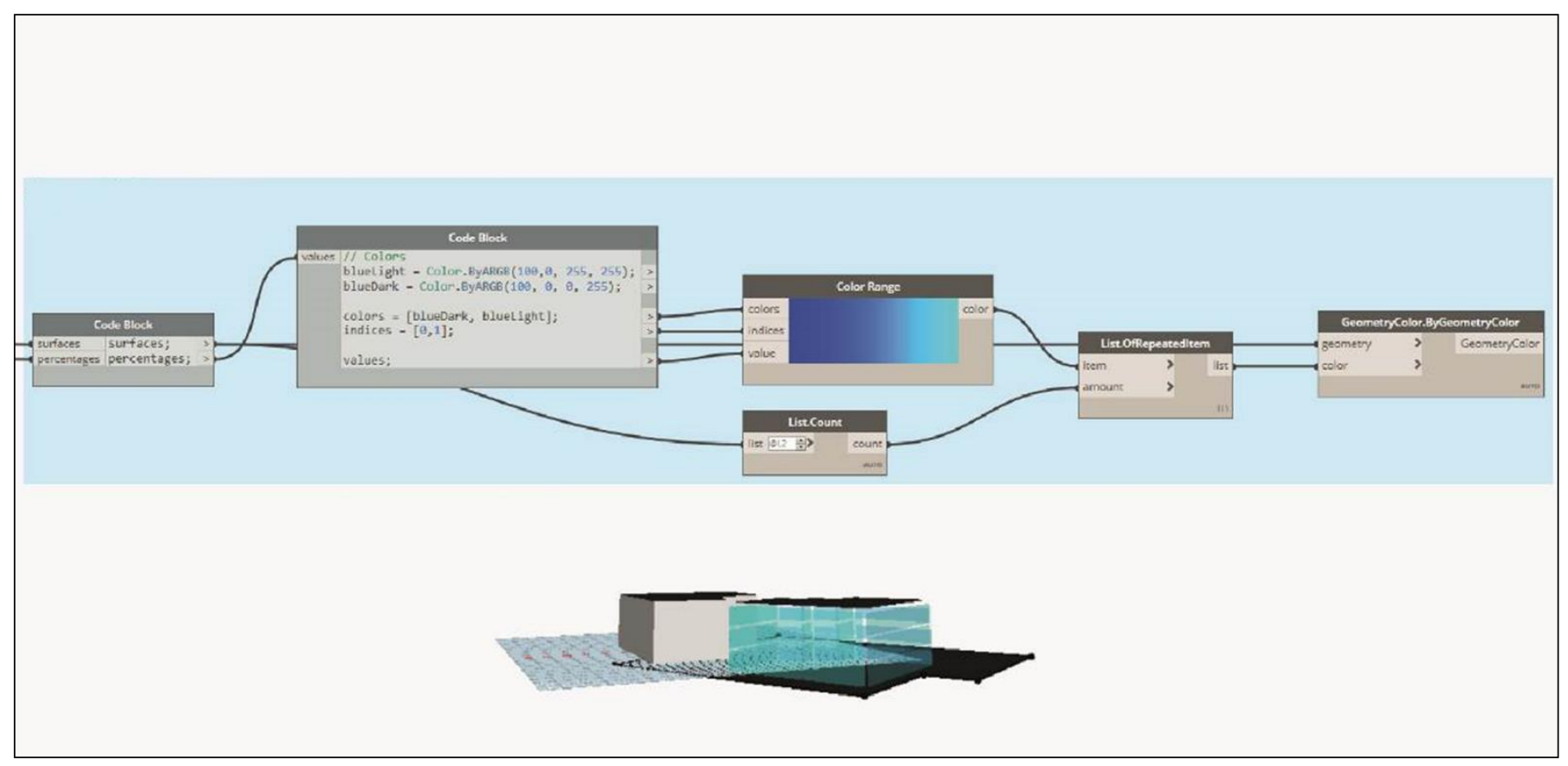

Fig. 2 Result of glazing surface. 
collect the information needed for analysis. Consequently, each iteration may take some time to be performed; moreover, often, the analysis may not be performed correctly and/or with inefficient rototranslations that would lead to place the building itself beyond the boundaries of the site under study.

Trivially, in this case, the following additional inputs and constraints are defined in order to obtain new and more optimal outputs:

- Site boundary, the site boundary lines from the Revit model (model curves) should be selected;

- Surrounding buildings, select the surrounding context that will influence the solar analysis;

- Volume, the mass (building), defined by the previous graphs (which will be repositioned);

- Site offset, a value that defines the offset from the site boundary;

- Average interstorey height, used for "Solar Analysis";

- Location coordinates, these are the coordinates used for Solar Analysis;

- Building rotation (in degrees), the angle between $0^{\circ}$ and $360^{\circ}$ by which the building will rotate from its initial position;

- U value, the parameter of displacement of the building within site along a given axis;

- V value, parameter of displacement of the building inside the site along the axis perpendicular to $\mathrm{U}$;

- Glazed surface, value in percentage of glazed surface, depending on orientation. (Fig. 3)

Once all these values are set, we continue with the generation of functions using Dynamo nodes. The script comprises a series of functions divided into groups within the graph; each group will have a name and a brief description. The name will indicate the type of function being executed, and the description will explain the related process in more detail.

The graph uses the Revit mass (or building) and extracts the geometry into Dynamo. The generator in this script provides a new position (based on the $\mathrm{U}$ and $\mathrm{V}$ values), along with a new rotation. The building is then moved to the new position point and rotated to fit the new angle. Once the building is in its new position relative to the site boundary, the solar analysis takes

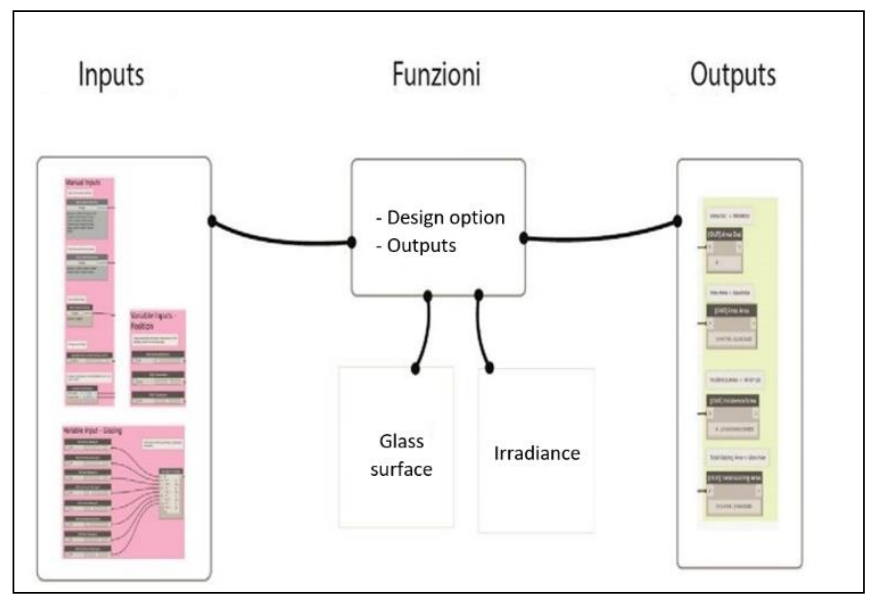

Fig. 3 Workflow. place, reviewing all exterior vertical surfaces of the building and calculating their solar incidence. To complete the Dynamo file with the contextual information, Formit was used, which was helpful to take advantage of the speed of mass modelling and the quick link with Bing satellite maps.

So, after having modelled in masses the volumes of neighbouring structures, the file is saved in Formit format and exported to Revit (to take advantage of the potential of Generative Design, it is necessary to work in Dynamo, which is integrated with Revit since version 2020). Once the file has been exported with the volume in Revit, the latter recognizes the Formit masses in families in Revit automatically. At this stage, the lot used as the "Site Offset" constraint is delimited. Having defined the geometry in Dynamo, it is often necessary to use other auxiliary geometries to facilitate the overall process. Note that all unnecessary geometry is disabled in Dynamo, to ensure that the displayed geometry shows only the final geometry output. Thus, all nodes with the preview disabled will not make the output visible in Generative Design. In this case, only the main building and the resulting solar analysis will be visible.

The solar analysis is represented on the exterior surfaces of the building as coloured grid points. These vary in colour from yellow to red, where yellow indicates low incidence and red indicates high incidence. Similarly, to display the corresponding glazed area, a scale of blue was set with the following parameters:

- Out area $\left(\mathrm{m}^{2}\right)$, area of the building that will be outside the site boundary;

- Free area $\left(\mathrm{m}^{2}\right)$, area of the inner boundary of the site that is not occupied by the base of the building;

- Average incidence $\left(\mathrm{m}^{2}\right)$, the average incidence of the exterior walls of the building. (Fig. 2)

If "area out" is greater than 0 , the options are invalid, and all outputs are penalized according to whether they should be enlarged or minimized, and likewise, all null results will not be displayed and therefore not used.

So, having set the inputs and constraints, created the Dynamo graph, and chosen the outputs needed to discretize the results of the design problem, it is possible to move on to the development phase in Generative Design.

To export the work from Dynamo to Refinery, it was enough to save the project and export it to Refinery. In this phase, according to the choice and the need of the designer, the Generative Design development is set: Randomize, Optimize, Cross Product, and Like this. Once the study is complete, the results can be explored through the tables and charts in Refinery's "Explore Results" dialog box. Finally, in the Results View phase, it is possible to choose from the results obtained and then refine the optimal result with the settings chosen in Dynamo to continue the design in the BIM environment and complete the project in the defined Level of Development.

Generalizing and without automating the process of coding design options, that is, not running these scripts in Dynamo, the user would have to intervene 'manually' on the building until he finally managed to find the desired solutions. This process, 
besides being more time consuming, is in any case less constrained by verifications.

Since the goal in this example was to define the best position and rotation for the minimum or maximum solar incidence, it was possible to use the "Optimize" method: larger site offset values would limit the space the building can move and thus also reduce the risk of it falling outside the site boundaries.

\section{CONCLUSION}

This paper proposed implementing a generative design workflow to analyse, in the first instance, the impact of radiation on a building, based on the placement of a building on the site and its surroundings. The course shows how the Generative Design process can result in good design strategies while revealing higher-level insights into the potential conflicts and trade-offs between design goals.

It was possible to highlight how at the base of Generative Design, there is always the choice of inputs and constraints that do not take responsibility away from the engineer and/or architect, but that can be gradually refined by further improving the generated strategies and leading to a more informed design. The Generative Design could also extend its potential to meet stakeholder needs for living comfort (temperature, air quality, acoustics, etc.), energy comfort, and general well-being. This can be achieved by extending the process to the evaluative component of generative design for architectural space planning and describing a set of new metrics for the automatic assessment of end-user satisfaction within spaces defined [26]. Machine learning can thus be a great tool to complement Generative Design when large datasets are available. Technically, we could try to leverage Machine Learning at any stage of the generative design process.

\section{REFERENCES}

[1] S. Abrishami, J. S. Goulding, F. P. Rahimian, and A. Ganah, "Integration of BIM and generative design to exploit AEC conceptual design innovation," Journal of Information Technology in Construction, vol. 19, 2014.

[2] I. Caetano and A. Leitão, "Integration of an algorithmic BIM approach in a traditional architecture studio," Journal of Computational Design and Engineering, vol. 6, no. 3, 2019, doi: 10.1016/j.jcde.2018.11.004.

[3] G. D'Aniello, M. Gaeta, F. Orciuoli, G. Sansonetti, and F. Sorgente, "Knowledge-Based Smart City Service System," Electronics, vol. 9, no. 6, Jun. 2020, doi: 10.3390/electronics9060965.

[4] F. Colace, M. de Santo, M. Lombardi, F. Pascale, D. Santaniello, and A. Tucker, "A Multilevel Graph Approach for Predicting Bicycle Usage in London Area," in Fourth International Congress on Information and Communication Technology. Advances in Intelligent Systems and Computing, vol 1027, vol. 1027, Y. XS., S. S., D. N., and J. A, Eds. Springer, Singapore, 2020, pp. 353-362. doi: 10.1007/978981-32-9343-4_28.

[5] F. Colace, D. Santaniello, M. Casillo, and F. Clarizia, "BeCAMS: A behaviour context aware monitoring system," 2017. doi: 10.1109/IWMN.2017.8078374.

[6] M. Carratu, M. Ferro, A. Pietrosanto, P. Sommella, and V. Paciello, "A Smart Wireless Sensor Network for PM10 Measurement," in 2019 IEEE International Symposium on Measurements and Networking, $M$ and N 2019 - Proceedings, Jul. 2019, pp. 1-6. doi: 10.1109/IWMN.2019.8805015.

[7] R. Guida, M. C. de Simone, P. Dašić, and D. Guida, "Modeling techniques for kinematic analysis of a six-axis robotic arm," IOP
Conference Series: Materials Science and Engineering, vol. 568, Sep. 2019, doi: 10.1088/1757-899X/568/1/012115.

[8] F. Colace, M. Lombardi, F. Pascale, and D. Santaniello, "A Multilevel Graph Representation for Big Data Interpretation in Real Scenarios," in 2018 3rd International Conference on System Reliability and Safety (ICSRS), Nov. 2018, pp. 40-47. doi: 10.1109/ICSRS.2018.8688834.

[9] C. Esposito, A. Castiglione, F. Palmieri, and A. de Santis, "Integrity for an Event Notification Within the Industrial Internet of Things by Using Group Signatures," IEEE Transactions on Industrial Informatics, vol. 14, no. 8, Aug. 2018, doi: 10.1109/TII.2018.2791956.

[10] F. Colace, M. Lombardi, F. Pascale, and D. Santaniello, "A multilevel approach for forecasting critical events in smart cities," 2018. doi: 10.18293/DMSVIVA2018-002.

[11] A. Castiglione et al., "Securing the internet of vehicles through lightweight block ciphers," Pattern Recognition Letters, vol. 135, pp. 264-270, Jul. 2020, doi: 10.1016/j.patrec.2020.04.038.

[12] F. Tao and M. Zhang, "Digital Twin Shop-Floor: A New Shop-Floor Paradigm Towards Smart Manufacturing," IEEE Access, vol. 5, 2017, doi: 10.1109/ACCESS.2017.2756069.

[13] J. O. Blosiu, "Use of synectics as an idea seeding technique to enhance design creativity," in Proceedings of the IEEE International Conference on Systems, Man and Cybernetics, 1999, vol. 3. doi: 10.1109/icsmc.1999.823365.

[14] Y. S. Kim, "Personal characteristics and design-related performances in a creative engineering design course," 2003.

[15] RIBA, BIM Overlay to the RIBA Outline Plan of Work. 2012.

[16] T. Fischer and C. Herr, "Teaching generative design," ... of the 4th Conference on Generative Art, 2001.

[17] C. M. Herr, "Generative architectural design and complexity theory," 2002.

[18] J. Frazer, J. Frazer, L. I. U. Xiyu, T. Mingxi, and P. Janssen, "Generative and Evolutionary Techniques for Building Envelope Design," 5th International Generative Art Conference GA2002, vol. 2002, no. December, 2002.

[19] J. Krause, "Reflections: The Creative Process of Generative Design in Architecture," Generative Arts Conference, 2003.

[20] S. Abrishami, J. Goulding, F. Pour Rahimian, and A. Ganah, "Virtual generative BIM workspace for maximising AEC conceptual design innovation: A paradigm of future opportunities," Construction Innovation, vol. 15, no. 1, 2015, doi: 10.1108/CI-07-2014-0036.

[21] A. Chaszar and S. C. Joyce, "Generating freedom: Questions of flexibility in digital design and architectural computation," International Journal of Architectural Computing, vol. 14, no. 2, 2016, doi: 10.1177/1478077116638945.

[22] F. a Bukhari, "A Hierarchical Evolutionary Algorithmic Design (HEAD) System for Generating and Evolving Building Design Models," 2011.

[23] P. Zhang and W. Xu, "Quasicrystal structure inspired spatial tessellation in generative design," in CAADRIA 2018 - 23rd International Conference on Computer-Aided Architectural Design Research in Asia: Learning, Prototyping and Adapting, 2018, vol. 1. “Autodesk Refinery program.” Accessed: May 31, 2021. [Online]. Available: www.autodesk.com/solutions/generativedesign/architecture-engineering-construction

[25] "Generative Design and Dynamo Primer." Accessed: May 31, 2021. [Online]. Available: https://www.generativedesign.org/

[26] L. Villaggi, J. Stoddart, D. Nagy, and D. Benjamin, "Survey-Based Simulation of User Satisfaction for Generative Design in Architecture," in Humanizing Digital Reality, 2018. doi: 10.1007/978-981-10-6611-5_36. 